\title{
Centros de simulación quirúrgica regionales y certificación a distancia (telesimulación). Una innovación pionera en el mundo conducida por la Sociedad de Cirujanos de Chile
}

\author{
Regional surgical simulation centers and remote certification \\ (telesimulation). A pioneering innovation in the world conducted \\ by the Society of Surgeons of Chile
}

Nicolás Jarufe ${ }^{1}$, Marcelo Barra² y Julián $\operatorname{Varas}^{3}$

Uno de los principales avances que ha tenido la cirugía en los últimos años, ha sido el desarrollo de las intervenciones mini-invasivas. Este tipo de procedimientos está siendo utilizado en la mayoría de las especialidades quirúrgicas, pero es especialmente relevante en la cirugía del aparato digestivo. $\mathrm{Si}$ bien las operaciones de vesícula se realizan por laparoscopía con éxito hace décadas, hoy en día se utiliza en casos tan complejos como gastrectomías por cáncer gástrico, cirugías de la obesidad, páncreas, resecciones de hígado, colon y recto, etc. Sus resultados son al menos comparables con la vía tradicional o abierta, pero con claras ventajas en la recuperación de los pacientes con lo que se logra menos dolor posoperatorio, menos días de hospitalización y se acorta el período de reintegro laboral, además de los claros beneficios estéticos. Ya están apareciendo reportes donde se comprueba incluso mejores resultados en morbimortalidad para algunos procedimientos gracias al menor volumen de sangrado descrito para la cirugía laparoscópica. En casos de pacientes oncológicos, estos se encontrarían en mejores condiciones y de manera más rápida, disponibles para el inicio de quimioterapia adyuvante de ser necesario.

Todas estas bondades descritas de la cirugía mini-invasiva, contrasta con la realidad no sólo nacional sino también en grandes centros americanos y europeos, donde ha sido difícil de implementar y, por sobre todo, difícil de entrenar, debido en parte a su larga curva de aprendizaje. El modelo mentoraprendiz, utilizado clásicamente en la enseñanza y entrenamiento quirúrgico, es muy dependiente de variables como la capacidad, calidad y experiencia de tutores donde pueden existir dramáticas diferencias que hacen disímil la transferencia del aprendi- zaje a los alumnos. Si a lo anterior agregamos las restricciones al horario laboral de los residentes, el elevado costo en sus formaciones y por supuesto el tener que otorgar máxima seguridad y calidad de atención a los pacientes, tenemos como consecuencia la menor exposición de los residentes a la sala operatoria y a casos complejos.

En una encuesta realizada en USA a los directores de programa de especialización quirúrgica, aparece que el $21 \%$ de ellos cree que los residentes no están preparados para el pabellón, un 38\% demuestran falta de liderazgo frente al paciente, un $26 \%$ es incapaz de reconocer los planos anatómicos y el $66 \%$ es incapaz de operar sin supervisión durante más de $30 \mathrm{~min}$. En lo que respecta a cirugía mini-invasiva, el 30\% no puede manipular el tejido en forma atraumática, un $56 \%$ es incapaz de suturar por laparoscopía y un 30\% no es capaz de realizar una colecistectomía laparoscópica. Estas cifras no debieran diferir de nuestra realidad, las que incluso podrían ser peores.

La simulación quirúrgica por otra parte ha demostrado claros beneficios en adquirir habilidades carentes y acortar curvas de aprendizaje. Progresivamente se han ido desarrollando modelos de entrenamiento simulado en cirugía desde los básicos a los más avanzados que involucran anastomosis intracorpóreas intestinales con escalas globales y específicas de evaluación que han sido definidas y validadas en la literatura. También existen modelos para cirugías de subespecialidad como la colorrectal, esófago-gástrica, hígado y páncreas entre otras. La aplicación de los modelos de entrenamiento simulado requiere no solamente de alta inversión tecnológica sino tal vez, lo más importante y difícil de lograr es la capacidad docente para evaluar a los
'Presidente $\mathrm{SOCICH}$.

2Vice-Presidente SOCICH. ${ }^{3}$ Coordinador de Centros Regionales de Simulación.

Correspondencia a: Dr. Nicolas Jarufe njarufe@med.puc.cl 
residentes y cirujanos, y poder acreditarlos en la medida que cumplen los requisitos de los entrenamientos. Existen instituciones universitarias en Santiago que han logrado reunir un número adecuado de docentes entrenados y capacitados para certificar los alumnos de programa de simulación. En regiones sin embargo, a pesar de contar algunas de ellas con simuladores, no cuentan con los docentes especializados que puedan acreditar las distintas etapas del entrenamiento. Considerando estos argumentos, la Sociedad de Cirujanos de Chile (SOCICH) ha desarrollado un plan de promover la simulación y llevarla a la mayoría de las residencias quirúrgicas del país, de manera de asegurar las competencias entregadas a los residentes, además, de incentivar la realización de cursos teóricos y prácticos ("handson") que pueden traer beneficios académicos y económicos a los Centros que la realicen.

A través de la utilización de tecnología de aprendizaje y una aplicación (Lapp) especialmente diseñada, los alumnos de regiones podrán realizar sus entrenamientos en cursos básicos, avanzados y posteriormente, de subespecialidad; subir sus vídeos del ejercicio realizado a esta aplicación la cual notificará al docente quien a su vez enviará feedback del entrenamiento y vídeos con instrucciones para corregir los errores y, finalmente, tener la certificación validada por la SOCICH y la Pontificia Universidad Católica de Chile. A futuro se incorporarán otras Instituciones Universitarias a la acreditación y la idea es ir capacitando docentes regionales también de manera de crear autonomía en los Centros.
Esta iniciativa de la SOCICH cuenta con un importante apoyo de la industria así como de fondos obtenidos a través de concursos ganados con el proyecto tanto en Chile como en USA. Los aportes totales por Centro, superan los USD\$ 95.000 que serán utilizados en simuladores con sus respectivas pantallas HD, insumos quirúrgicos tales como generadores de energía, suturas mecánicas, trócares, pinzas, tijeras, etc. además de grabadores de video, tarjetas de memoria, set de modelos sintéticos y licencias para cursos básicos y avanzados. A lo anterior, se suman las inversiones que cada Centro realizará de manera de contar con la infraestructura necesaria: espacio físico, condiciones higiénicas, facilidades para los alumnos, lavaderos con agua fría y caliente para preparación de intestino y tejido ex-vivo e internet de banda ancha de al menos 50 mbps.

El Directorio de la SOCICH aprobó la ejecución de este importante desafío para 6 Centros regionales, 3 el 2018 y otros 3 el 2019. Este año se debiera inaugurar los Centros de la Universidad Católica del Norte en Coquimbo, Universidad de Concepción y Universidad Austral en Valdivia. Se entrenarán como piloto al menos 10 alumnos por Centro de manera de ir perfeccionando los procesos para su implementación definitiva. Será decisión del Presidente y su Directorio cuáles serán los Centros para el 2019 dependiendo de las condiciones locales y el interés que manifiesten de ser parte de esta gran iniciativa que, sin duda, contribuirá al desarrollo de nuestros cirujanos y la cirugía chilena.

\section{Bibliografía}

1. Mattar SG, Alseidi AA, Jones DB, Jeyarajah DR, Swanstrom LL, Aye RW, et al. General Surgery Residency inadequately Prepares Trainees for Fellowship: Results of a Survey of Fellowship Program Directors. Ann Surg. 2013;258:440-9.

2. Jarufe N, Martínez J. Rol de la Sociedad de Cirujanos de Chile en la Formación de Cirujanos Generales. Rev Chil Cir. 2015;67:578-9.

3. Jarufe N, Barra M. Comenzando un nuevo período con Énfasis en la Educación
Quirúrgica y las Regiones. Rev Chil Cir 2017;69:103-4.

4. Achurra P, Lagos A, Ávila R, Tejos R, Buckel E, Alvarado J, et al. Allowing New Opportunities in Advanced Laparoscopy Training Using a Full High Definition Training Box. Surgical Innovation 2017;24:66-71.

5. Castillo R, Buckel E, León F, Varas J, Alvarado J, Achurra P, et al. Effectiveness of Learning Advanced Laparoscopic Skills in a Brief Intensive. Laparoscopy Training Program. Journal of Surgical Education 2015;72:648-53.

6. Varas J, Buckel E, Crovari F, Pimentel F,
Martínez J, Jarufe, et al. Simulación en cirugía laparoscópica. Cirugía Española 2015;93:4-11.

7. Boza C, León F, Buckel E, Riquelme A, Crovari F, Martínez J, et al. Simulationtrained Junior Residents Perform Better than General Surgeons on Advanced Laparoscopic Cases. Surgical Endoscopy 2017;31:135-41.

8. Castillo R, Alvarado J, Moreno JP, Billeke P, Martínez C, Varas J, et al. Validation of a Visual-Spatial Secondary Task to Assess Automaticity in Laparoscopic Skills. Journal of Surgical Education 2017;101:15. 